\title{
SIMULATION OF SYNTHETIC JETS IN QUIESCENT AIR USING UNSTEADY REYNOLDS AVERAGED NAVIER-STOKES EQUATIONS
}

\author{
Veer N. Vatsa* \\ NASA Langley Research Center, Hampton, VA \\ Eli Turkel ${ }^{\dagger}$ \\ Tel-Aviv University, Israel and NIA, Hampton, VA
}

\section{Abstract}

We report research experience in applying an Unsteady Reynolds-Averaged Navier-Stokes (URANS) solver for the prediction of time-dependent flows in the presence of an active flow control device. The configuration under consideration is a synthetic jet created by a single diaphragm piezoelectric actuator in quiescent air. Time-averaged and instantaneous data for this case were obtained at Langley Research Center, using multiple measurement techniques. Computational results for this case using one-equation Spalart-Allmaras and two-equation Menter's turbulence models are presented here along with comparisons with the experimental data. The effect of grid refinement, preconditioning and time-step variation are also examined.

\section{Introduction}

Significant interest has been growing in the aerospace community in the field of flow control in recent years. An entire AIAA conference is now devoted every other year to this field. In particular, an international workshop, CFDVAL2004 [1], was held in March 2004 at NASA Langley Research Center to assess the state-of-the-art for measuring and computing aerodynamic flows in the presence of synthetic jets. Thomas et al. [2] have conducted an exhaustive and comprehensive survey identifying the feasibility of using active flow control to improve the performance of both external and internal flows. Suggested applications cover a wide range from smart materials and micro-electro-mechanical systems (MEMS) to synthetic zero net mass jets for enhancing control forces, reducing drag, increasing lift and enhancing mixing to name a few. It is also conjectured that active flow control would permit the use of thicker wing sections in non-conventional configurations, such as the Blended Wing Body (BWB) configuration without compromising the aerodynamic performance.

Most of the research in the area of active flow control is of empirical nature, mainly due to the cost and lack of confidence in computational methods for such complex flows. However, without the availability of efficient and well-calibrated computational tools, it will be a very difficult, expensive and slow process to determine the optimum layout and placement for active flow control devices in practical applications. With the continuous reduction of computer costs in recent years, more attention is being devoted to the simulation of such unsteady flows, and many researchers have recently started examining various flow control devices from a computational point of view (Refs. [3] - [8]). With few exceptions, most of the numerical studies are undertaken without an active interaction with experimental investigators. Comparisons with experimental data are sometimes done years after the experimental data have been acquired. Under such a scenario, one has to reconstruct some of the details about the experimental arrangement and boundary conditions without the benefit of concrete and consistent information. Based on our experience from previous validation exercises [9], we recognized the need for active collaboration of the computational and experimental research. Without a symbiotic relationship among such groups, major misunderstandings can develop when

\footnotetext{
* Senior Member

${ }^{\dagger}$ Professor, Department of Mathematics, Associate Fellow
} 
results from these disciplines indicate significant differences. We were very fortunate to have a frank and cooperative relationship with the researchers conducting the experiments as well as access to pertinent experimental data.

Our primary objective for this work is to calibrate an existing computational scheme with experimental data for timedependent flows encountered in active flow control environments. Special attention is devoted to establish appropriate and stable boundary conditions for such flows, especially in the absence of detailed experimental data required for closure.

The configuration chosen for CFD validation is identified as Case 1 in the CFDVAL2004 workshop [1], and represents an isolated synthetic jet formed by a single diaphragm piezoelectric actuator exhausting into ambient quiescent air. Multiple measurement techniques including PIV, LDV and hot-wire probes were used to generate a large body of experimental data for this configuration. The details of the experimental setup and geometric configuration are described in Refs. [1, 10]. In this paper, we assess the effects of grid refinement, preconditioning and turbulence models on the flow field generated by this synthetic jet flow control device. We replace the actuator cavity with a simpler configuration. We demonstrate and calibrate our computational method for simulating synthetic jets by comparing the numerical results with the experimental data.

\section{Governing Equations}

A generalized form of the thin-layer Navier-Stokes equations is used to model the flow. The equation set is obtained from the complete Navier-Stokes equations by retaining the viscous diffusion terms normal to the solid surfaces in every coordinate direction. For a body-fitted coordinate system $(\xi, \eta, \zeta)$ fixed in time, these equations can be written in the conservative form as:

$$
V o l \frac{\partial(\mathbf{U})}{\partial t}+\frac{\partial\left(\mathbf{F}-\mathbf{F}_{\mathbf{v}}\right)}{\partial \xi}+\frac{\partial\left(\mathbf{G}-\mathbf{G}_{\mathbf{v}}\right)}{\partial \eta}+\frac{\partial\left(\mathbf{H}-\mathbf{H}_{\mathbf{v}}\right)}{\partial \zeta}=0
$$

where $\mathbf{U}$ represents the conserved variable vector. The vectors $\mathbf{F}, \mathbf{G}, \mathbf{H}$, and $\mathbf{F}_{\mathbf{v}}, \mathbf{G}_{\mathbf{v}}, \mathbf{H}_{\mathbf{v}}$ represent the convective and diffusive fluxes in the three transformed coordinate directions, respectively. In Eqn. (1), Vol represents the cell-volume or the Jacobian of the coordinate transformation. A multigrid-based, general purpose multi-block structured grid approach is used for the solution of the governing equations. In particular, the TLNS3D flow code is used in this study to solve Eqn. (1). Several references exist describing the discretization and algorithmic details of TLNS3D code. We include only a brief summary of the general features, and refer to the work of Vatsa and co-workers $[11,12]$ for further details regarding the TLNS3D code.

\section{Spatial Discretization}

The spatial terms in Eqn. (1) are discretized using a cell-centered finite volume scheme. The convection terms are discretized using second-order central differences with matrix artificial dissipation (second- and fourth- difference dissipation) added to suppress the odd-even decoupling and oscillations in the vicinity of shock waves and stagnation points $[13,14,15]$. The viscous terms are discretized with second-order accurate central difference formulas [11]. The zero-equation model of Baldwin-Lomax [16], one-equation model of Spalart-Allmaras [17] and Menter's two-equation SST model [18] are available in TLNS3D code for simulating turbulent flows. For the present computations, the Spalart-Allmaras (SA) model and the Menter's SST model are used for simulating turbulent flows.

\section{Temporal Discretization}

Regrouping the terms on right hand side into convective and diffusive terms, Eqn. (1) can be rewritten as:

$$
\frac{d \mathbf{U}}{d t}=-C(\mathbf{U})+D_{p}(\mathbf{U})+D_{a}(\mathbf{U})
$$

where $C(\mathbf{U}), D_{p}(\mathbf{U})$, and $D_{a}(\mathbf{U})$ are the convection, physical diffusion, and artificial diffusion terms, respectively. The cell-volume or the Jacobian of the coordinate transformation is included in these terms.

The time-derivative term can be approximated to any desired order of accuracy by a Taylor series

$$
\frac{d \mathbf{U}}{d t}=\frac{1}{\Delta t}\left[a_{0} \mathbf{U}^{n+1}+a_{1} \mathbf{U}^{n}+a_{2} \mathbf{U}^{n-1}+a_{3} \mathbf{U}^{n-2}+\ldots\right]
$$


The superscript $\mathrm{n}$ represents the last time level at which the solution is known, and $\mathrm{n}+1$ refers to the next time level to which the solution will be advanced. Similarly, n-1 refers to the solution at one time level before the current solution. Eqn. (3) represents a generalized backward difference scheme (BDF) in time, where the order of accuracy is determined by the choice of coefficients $a_{0}, a_{1}, a_{2} \ldots$ etc. For example, $a_{0}=1.5, a_{1}=-2$ and $a_{2}=.5$, results in a second order accurate scheme (BDF2) in time, which is the primary scheme chosen for this work due to its robustness and stability properties [19]. Regrouping the time-dependent terms and the original steady-state operator leads to the equation:

$$
\frac{a_{0}}{\Delta t} \mathbf{U}^{n+1}+\frac{E\left(\mathbf{U}^{n, n-1, . .}\right)}{\Delta t}=S\left(\mathbf{U}^{n}\right)
$$

where $E\left(\mathbf{U}^{n, n-1, . .}\right)$ depends only on the solution vector at time levels $\mathrm{n}$ and earlier. S represents the steady state operator or the right hand side of Eqn.(2). By adding a pseudo-time term, we can rewrite the above equation as:

$$
\frac{\partial \mathbf{U}}{\partial \tau}+\frac{a_{0}}{\Delta t} \mathbf{U}^{n+1}+\frac{E\left(\mathbf{U}^{n, n-1, . .}\right)}{\Delta t}=S\left(\mathbf{U}^{n}\right)
$$

\section{Solution Algorithm}

The algorithm for solving unsteady flow relies on the steady-state algorithm in the TLNS3D code [11, 12]. The basic algorithm consists of a five-stage Runge-Kutta time-stepping scheme for advancing the solution in pseudo-time, until the solution converges to a steady state. Efficiency of this algorithm is enhanced through the use of local time-stepping, residual smoothing and multigrid techniques developed for solving steady-state equations. Because the Mach number in much of the domain is very low we consider the use of preconditioning methods [26, 27].

In order to solve the time-dependent Navier-Stokes equations (Eqn. 5), we add another iteration loop in physical time outside the pseudo-time iteration loop in TLNS3D. For fixed values of $S\left(\mathbf{U}^{n}\right), E\left(\mathbf{U}^{n, n-1, . .}\right)$, we iterate on $\mathbf{U}^{n+1}$ using the standard multigrid procedure of TLNS3D developed for steady-state, until the desired level of convergence is achieved. This strategy, originally proposed by Jameson [20] for Euler equations and adapted for the TLNS3D viscous flow solver by Melson et. al [19], is popularly known as the dual time-stepping scheme for solving unsteady flows. The process is repeated until the desired number of time-steps have been completed. The details of the TLNS3D flow code for solving unsteady flows are available in Refs. [19, 25, 28].

\section{Boundary Conditions}

The boundary conditions required for solving the Navier-Stokes equations, such as the no-slip, no injection, fixed wall temperature or adiabatic wall, far-field and extrapolation conditions are well understood and readily available in most flow codes including the TLNS3D code. The most accurate procedure to simulate the moving diaphragm would require moving grid capability. For simplicity, we chose to simulate this type of boundary condition by a periodic velocity transpiration condition. The frequency of the transpiration velocity at the diaphragm surface in the numerical simulation corresponds to the frequency of the oscillating diaphragm. The peak velocity at the diaphragm surface was obtained from numerical iteration to match the experimentally measured peak velocity of the synthetic jet emanating from the slot exit. The pressure at the moving diaphragm is also required for closure. However, in the absence of unsteady pressure data from the experiment, we imposed a zero pressure gradient at the diaphragm boundary. We also tested the pressure gradient boundary condition obtained from one-dimensional normal momentum equation [21], which had very little impact on the solutions. Due to the simplicity and robustness, we selected the zero pressure gradient boundary condition at the diaphragm surface.

\section{Synthetic Jet Test Case: Background}

The test configuration examined in this paper corresponds to a single diaphragm piezoelectric actuator operating in quiescent air. The oscillatory motion of the diaphragm produces a synthetic jet that exhausts into surrounding air. This configuration, shown in Fig. 1, consists of a $1.2 \mathrm{~mm}$ wide rectangular slot connected to a cavity with a piezoelectric diaphragm, and corresponds to case 1 of the CFDVAL2004 workshop on flow control devices [1]. The cavity and diaphragm geometry of this actuator are highly three-dimensional in the interior. However, the actual slot through which the fluid emerges is a high aspect ratio 
rectangular slot and can be modeled as a two-dimensional configuration. Partial view of the 2-D grid provided to the workshop participants, and used by the present authors is shown in Figs. 2(a) and 2(b). The computational results contributed by the workshop participants are available in refs. [1,22]. A consensus developed during the workshop that simulating the flow field inside the actuator cavity with an oscillating piezoelectric diaphragm from first principles was beyond the capability of the existing CFD codes. Most of the workshop participants, including the present authors modeled the internal cavity of the actuator as a two-dimensional configuration, and simulated the diaphragm motion via a transpiration condition imposed at the diaphragm surface. Some of the workshop participants further simplified the cavity modeling by imposing a transpiration condition at the bottom part of the slot's neck or even directly at the slot exit. After examining these results, we concluded that as long as the unsteady velocity signal at the slot exit replicates experimental conditions, details of the cavity modeling have an insignificant effect on the development of the synthetic jet emanating from the slot. Another conclusion derived from the CFDVAL2004 workshop was that no particular methodology or turbulence model emerged superior for simulating this test case $[1,22]$.

One of the major difficulties identified during the CFDVAL2004 workshop was the large disparity in experimental data obtained from PIV, hot-wire probes and LDV measurement techniques. Such a variation in experimental data made it difficult to validate the numerical methods. Part of the difficulty in acquiring a consistent set of experimental data arose from the fact that the performance of the piezoelectric diaphragm depends on ambient conditions. Therefore, its performance degrades over time which means that for a given input voltage, the actuator produces smaller jet velocities as it ages. Because these experiments were conducted over a period of several months, inconsistencies creeped in the data.

We include here sample results obtained with the TLNS3D code to encapsulate the status of CFD simulations for this configuration at the conclusion of the CFDVAL2004 workshop. For these computations, we used the 2-D grid of approximately 65,000 nodes from the CFDVAL2004 workshop website as the baseline grid. As depicted in Figs. 2(a), 2(b), this grid includes the internal cavity and the diaphragm geometry. The transpiration condition is imposed at the diaphragm surface, which is represented by the vertical line on the left side at the bottom of Fig. 2(b). We present the time-history of the (vertical) vvelocity at $x=0, y=0.12 \mathrm{~mm}$, and the time average of the v-velocity along the jet centerline in figures 3 and 4 , respectively. The computations were performed with several grid densities, two physical time steps and two turbulence models. The coarse grid (cg) was obtained by eliminating every other point from the baseline grid, and a fine grid (fg) was obtained by adding 50\% points in the normal direction. We see from these figures that varying all these factors did not produce any major changes in the computational results in region near the slot exit. However, further away from the slot exit, coarse grid (cg) results indicate that the coarser grid does not provide adequate resolution for this problem. A finer grid (fg) and a reduction in time step (low dt) had an insignificant effect on the computational results. Because of the scatter between the experimental PIV and hotwire data, it is difficult to make any definitive conclusion regarding the accuracy of turbulence models.

\section{Results}

Yao et. al [10] have recently revisited the synthetic jet test case and acquired experimental data for this configuration with a new diaphragm. The detailed field data was obtained with the PIV technique. In addition, pointwise data along the jet centerline was obtained with hotwire and LDV techniques. The performance of the actuator was monitored regularly. The results from this study show good consistency among multiple measurement techniques [10].

We simulated the new experimental test case with a simplified cavity geometry, shown schematically in Fig. 5. The transpiration condition is imposed at the bottom of the slot's neck to simulate the velocity generated by the oscillating diaphragm. Similar boundary condition treatment produced satisfactory results at the CFDVAL2004 workshop, and has also been studied in detail by Yamaleev and Carpenter [23]. They demonstrated that for actuators with deep cavities, specifying the transpiration condition at a distance of at least 4-5 slot widths away from the slot exit produces only a small loss in numerical accuracy. A top-hat velocity profile, with a dominant frequency of $450 \mathrm{~Hz}$., replicating the experimental conditions was imposed at the bottom boundary. The precise form of the velocity signal was obtained by curve fitting the measured velocities at the slot exit $(\mathrm{x}=0, \mathrm{y}=0.3 \mathrm{~mm})$ with a Fast Fourier Transform to reflect the proper mode shape and to ensure zero net mass transfer. The amplitude of this velocity was determined numerically to match the peak velocity from the experiment at the slot exit. The free stream Mach number in the exterior quiescent region is specified as $M_{\infty}=.001$ for simulating incompressible flow in the compressible flow code to avoid numerical difficulties at Mach zero.

The computational grid for this case was obtained from the baseline grid described in the previous section by eliminating the portions of grid below the neck of the slot. The resulting grid consists of over 60,000 nodes, and should provide adequate resolution based on the grid refinement study reported in reference [1], and as seen from the results in figures 3 and 4. Similarly, 
72 time-steps/period corresponding to $5^{\circ}$ phase angle between the time steps should provide adequate temporal resolution for this problem. The one-equation Spalart-Allmaras (SA) [17] turbulence model was used to simulate the effect of turbulence in these computations. Based on the peak jet velocity and slot width, the Reynolds number is approximately 3000, and therefore falls in the regime where the jet is expected to be turbulent. Therefore, the flow was assumed to be fully turbulent in the present simulations.

The time-history of the vertical velocity for a complete period from the computational results with SA model is compared with the experimental data in Fig. 6 at $x=0$ and $y=0.3 \mathrm{~mm}$. This is the closest point to the slot exit where the PIV data is available. In addition to the PIV data, LDV measurements are also available at this location and are shown here. The LDV data was scaled down by a factor of 0.9 as suggested by Yao et. al [10] to match the diaphragm displacement for the two sets of measurements, and is in good agreement with the PIV data except for a flatter region near the $150^{\circ}$ phase. The overall agreement between the computational and experimental results is quite good at this location, and therefore serves as an accurate boundary data for the jet emerging from the slot.

Next, we compare the time-averaged v-velocities along the jet centerline in Fig. 7 with the PIV and LDV data. The experimental data from two different techniques (PIV and LDV) is found to be in good agreement with each other, giving credibility to the accuracy and consistency of the measurements for this case. The overall agreement of the baseline TLNS3D results with the experimental data is also quite good. The low-speed preconditioning results included in this figure are essentially identical to the baseline results. Because low-speed preconditioning ([26]-[28]) primarily reduces the artificial viscosity for unsteady flows, it is inferred that the artificial viscosity is already low in these simulations. The time-averaged v-velocity profiles at $\mathrm{y}=1$ and $4 \mathrm{~mm}$ are shown in Fig. 8 and Fig. 9, respectively. Except for a smaller velocity peak at the centerline at $\mathrm{y}=1 \mathrm{~mm}$, the computational results are in very good agreement with the experimental data.

The contour plots of the time-averaged v-velocities based on PIV measurements covering a distance of $8 \mathrm{~mm}$ from slot exit are shown in Fig. 10(a). Note that the high resolution PIV data was limited up to a distance of $8 \mathrm{~mm}$ from slot exit because of cost and time constraints. The contour plots from our baseline computations are shown in Fig. 10(b) for comparison. The computational results appear to accurately capture all of the prominent features seen in the PIV data including the width and spreading rate of the synthetic jet.

We now examine the phase-averaged velocities at selected locations in space and time, starting with v-velocities at $\mathrm{y}=2$ and $4 \mathrm{~mm}$ along the jet center-line. The PIV and LDV data along with baseline TLNS3D solutions are shown at these locations in Figs. 11(a) and 11(b). The computational results are in fairly good agreement with the two sets of experimental data, especially in the suction phase. The agreement with the experimental data further away from the slot exit is slightly worse during the peak expulsion cycle. In particular, the CFD results predict a delayed phase shift for the peak expulsion, reflective of smaller convective speed for outward movement of the vortex compared to the experimental data.

We gain a broader perspective of the flow-field by examining the contour plots of the velocities at the phase angles representative of the expulsion (phase $=75^{\circ}$ ) and suction (phase $=255^{\circ}$ ) cycles. The velocity contours for streamwise (u-vel) and vertical velocities (v-vel) obtained from the PIV data and TLNS3D computational results are shown in Figs. 12 - 15 and Figs. 16 - 19. These figures were generated using identical contour levels for both the experimental and CFD data for providing a quantitative comparisons. Note that solid lines represent positive values, while dashed line represent negative values for the velocities. This sign convention is helpful in identifying the flow direction and the position of the vortex center. It is clear from these figures that the computational results capture most of the pertinent features observed experimentally and are in very good agreement for the suction phase. During the expulsion phase, the computed vortex center is located closer to the slot exit compared to experimental data, although the peak velocity at the vortex center is in good agreement with the PIV data. Yao et al. [10] have observed increasing three-dimensional effects for this case as one moves away from the slot exit, mainly due to ring vortices formed from the slot edges. We conjecture that these ring vortices induce forces that accelerate the convection of synthetic jet in the far field. Wheteher this indeed is the primary reason for differences in convective speed of the vortex, can only be verified by 3-D simulations with sufficient resolution to capture the vortical structure emanating from the edges of the slot.

\section{Concluding Remarks}

Detailed comparisons have been presented for time-averaged and phase-averaged velocities between experimental data and CFD results. The effect of truncation errors were found to be small based on a grid refinement, preconditioning and physical time-step refinement studies. The differences between the solutions obtained from the one-equation turbulence model of Spalart and two-equation model of Menter were found to be small in the near field for the synthetic jet. The modeling of the internal flow in the cavity of the actuator turned out be an extremely difficult problem. Fortunately, the development of the synthetic 
jet in the quiescent medium is driven primarily by the velocity field at the slot exit, and detailed modeling of the cavity is not warranted. Based on comparisons of the computational results with the original experimental data, it was decided to repeat the experiments to obtain more consistent data. The computational results in the reduced domain with a modified forcing function are found to be in much better agreement with the new experimental data in the near field. However, the agreement with the experimental data deteriorates in regions further away from the slot exit. Based on the available experimental data, it appears that the flow becomes three-dimensional after 5-6 slot widths away from the exit. Future work should focus on 3-D computations for this configuration to resolve such issues.

\section{Acknowledgment}

The authors would like to acknowledge Dr. C. Yao of NASA Langley research center for sharing his experimental data for the synthetic jet and for constructive discussions on the experimental procedures used for acquiring the data.

\section{References}

[1] CFD Validation of Synthetic Jets and Turbulent Separation Control: Langley Research Center Workshop, Willimasburg, VA, March 29-31, 2004. (http://cfdval2004.larc.nasa.gov)

[2] Thomas R.H.; Choudhri, M.L.; and Joslin, R.D.: "Flow and Noise Control: Review and Assessment of Future Directions". NASA TM under preparation.

[3] Hassan, A.A. and Mults, A.A.: "Transverse and Near-Tangent Synthetic Jets for Aerodynamic Flow Control". AIAA paper 2000-4334, Aug. 2000.

[4] Mittal, R., Rampunggoon, P.; and Udaykumar, H.S.: "Interaction of a Synthetic Jet with a Flat Plate Boundary Layer". AIAA paper 2001-2772.

[5] Lin, H. and Chieng, C.C.: "Computations of Compressible Synthetic Jet Flows Using Multigrid/Dual Time Stepping Algorithm”. AIAA Paper 99-3114.

[6] Donovan, J.F.; Kral, L.D.; and Cary, A.W.: "Active Flow Control Applied to an Airfoil”. AIAA Paper 98-0210, Jan. 1998.

[7] Kral, L.D.; Donoval, J.F.; Cain, A.B.; and Cary, A.W.: "Numerical Simulation of Synthetic Jet Actuators". AIAA Paper 97-1824, June-July 1997.

[8] Joslin, R.D.; Horta, L.G.; and Chen, F.J.: "Transitioning Active Flow Control to Applications". AIAA Paper 99-3575, June-July 1999.

[9] Viken, S.A.; Vatsa, V.N.; Rumsey, C.L.; and Carpenter, M.H.: "Flow Control Analysis on the Hump Model with RANS Tools". AIAA Paper 2003-218, Jan. 2003.

[10] Yao, C.; Chen, F.J.; Neuhart, D.; and Harris, J.: "Synthetic Jet Flow Field Database for CFD Validation". AIAA Paper 2004-2218, June 2004.

[11] Vatsa, V.N. and Wedan, B.W.: "Development of a multigrid code for 3-d Navier-Stokes equations and its application to a grid-refinement study". Computers and Fluids. 18:391-403. 1990.

[12] Vatsa, V.N.; Sanetrik, M.D.; and Parlette, E.B.: "Development of a Flexible and Efficient Multigrid-Based Multiblock Flow Solver”. AIAA paper 93-0677, Jan. 1993.

[13] Jameson, A.; Schmidt, W.; and Turkel, E.: "Numerical Solutions of the Euler Equations by Finite Volume Methods Using Runge-Kutta Time-Stepping Schemes”. AIAA Paper 1981-1259, June 1981.

[14] Turkel, E. and Vatsa, V.N.: "Effect of artificial viscosity on three-dimensional flow solutions". AIAA Journal, vol. 32, no. 1, Jan. 1994, pp. 39-45. 
[15] Swanson, R.C. and Turkel, E.: “On Central Difference and Upwind Schemes”. Journal of Computational Physics, vol. 101, 1992, pp. 292-306.

[16] Baldwin,B.S. and Lomax,H.: “Thin layer approximation and algebraic model for separated turbulent flows". AlAA Paper 78-257, Jan. 1978.

[17] Spalart, P.A. and Allmaras, S.R.:“A one-equation turbulence model for aerodynamic flows”. AIAA Paper 92-439, Reno, NV, Jan. 1992.

[18] Menter, F.R.: “Zonal Two Equation k- $\omega$ Turbulence Models for Aerodynamic Flows”. AIAA paper 93-2906, Orlando, Fl, 1993.

[19] Melson,N.D.; Sanetrik, M.D.; and Atkins, H.L.:"Time-accurate calculations with multigrid acceleration”. Appeared in "Proceedings of the Sixth Copper Mountain conference on multigrid methods", 1993, edited by: Melson, N.D.; Manteuffel, T.A.; and S.F. McCormick, S.F.

[20] Jameson, A.: “Time Dependent Calculations Using Multigrid, with Applications to Unsteady Flows past Airfoils and Wings". AIAA Paper 91-1596, 1991.

[21] Rizzetta, D.P.; Visbal. M.R.; and Stanek, M.J.: "Numerical Investigation of Synthetic Jet Flowfields". AIAA Paper 982910, June 1998.

[22] Rumsey, C.L.; Gatski, T.B.; Sellers III, W.L.; Vatsa, V.N.; and Viken, S.A.: "Summary of the 2004 CFD Validation Workshop on Synthetic Jets and Turbulent Separation Control”. AIAA Paper 2004-2217, June 2004.

[23] Yamaleev, N.K. and Carpenter, M.H.: "A Reduced Order Model for Efficient Simulation of Synthetic Jet Actuators". NASA TM-2003-212664, Dec. 2003.

[24] Yehoshua, T. and Seifert, A.: "Boundary Condition Effects on Oscillatory Momentum Generators”. AIAA Paper 03-3710, June 2003.

[25] Bijl, H.; Carpenter, M.H.; and Vatsa, V.N.: “Time Integration Schemes for the Unsteady Navier-Stokes Equations”. AIAA Paper 2001-2612.

[26] Turkel, E.: "Preconditioned Methods for Solving the Incompressible and Low Speed Compressible Equations". Journal of Computational Physics, vol 72 pp. 277-298, 1987.

[27] Turkel, E.: “ Preconditioning Techniques in Computational Fluid Dynamics”. Annual Reviews in Fluid Mechanics 1999, vol 31, pp. 385-416, 1999.

[28] Vatsa, V.N. and Turkel, E.: “Assessment of Local Preconditioners for steady state and time dependent flows". AIAA paper 2004-2134, June 2004. 


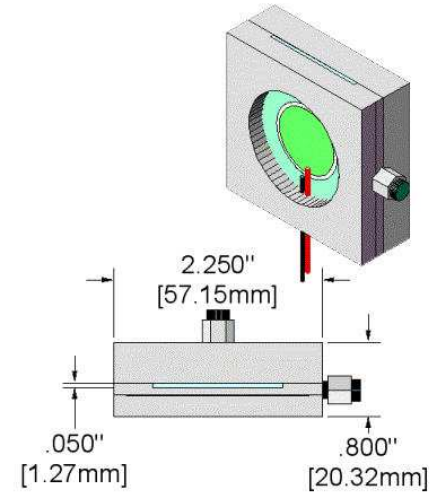

Figure 1: Schematic of Piezoelectric Actuator
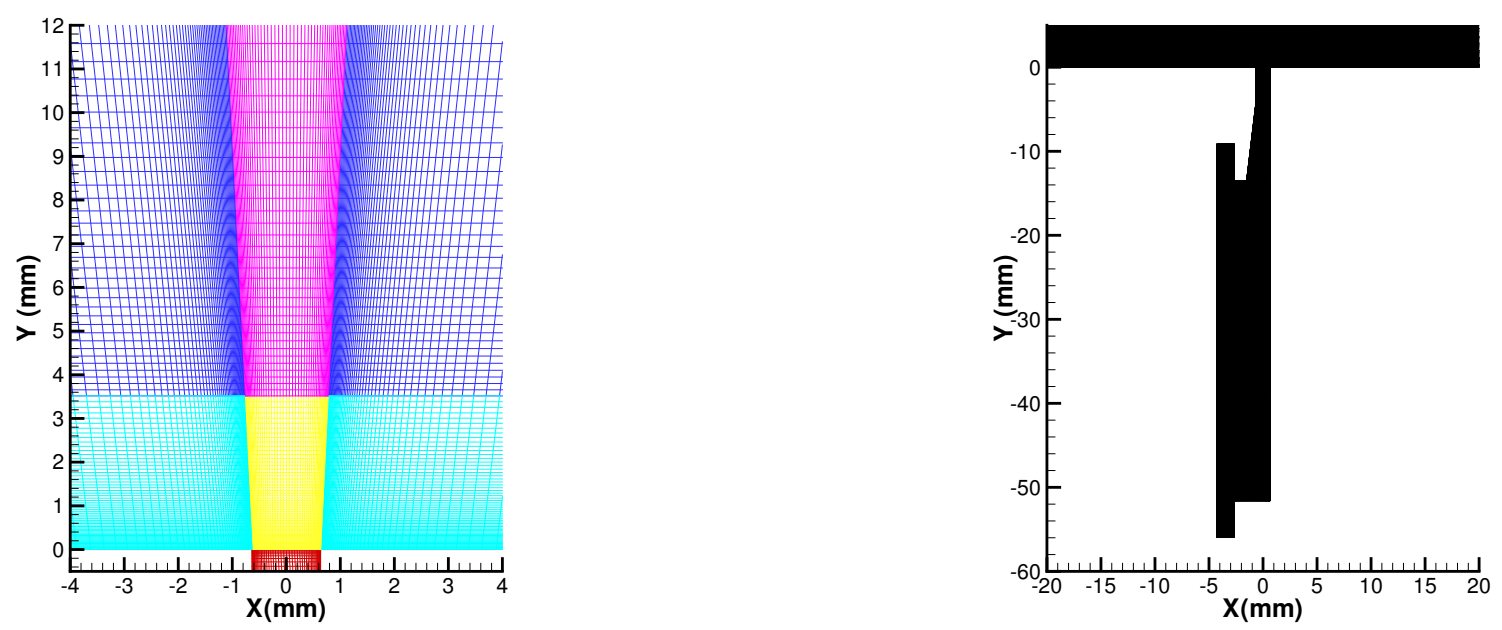

(a) Global view

(b) Detailed view

Figure 2: Computational grid for Piezoelectric Actuator 


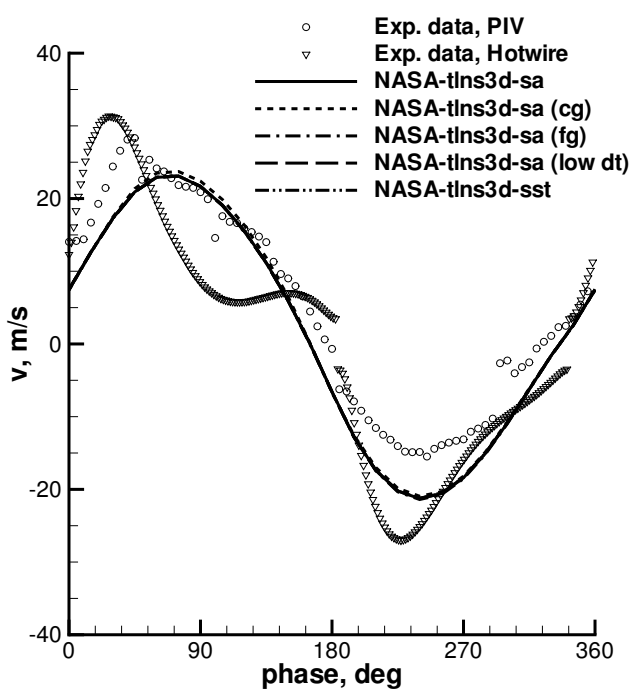

Figure 3: Time-history of v-velocity at $\mathrm{x}=0, \mathrm{y}=0.12$ $\mathrm{mm}$

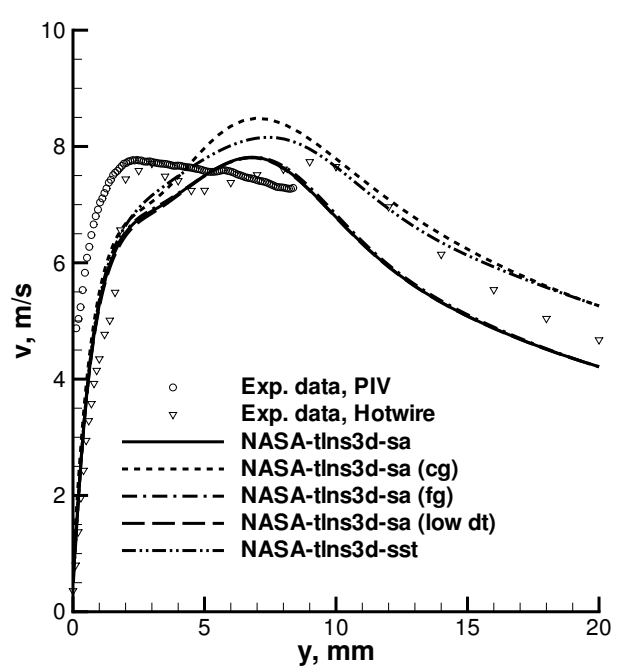

Figure 4: Time-averaged v-velocity along jet centerline

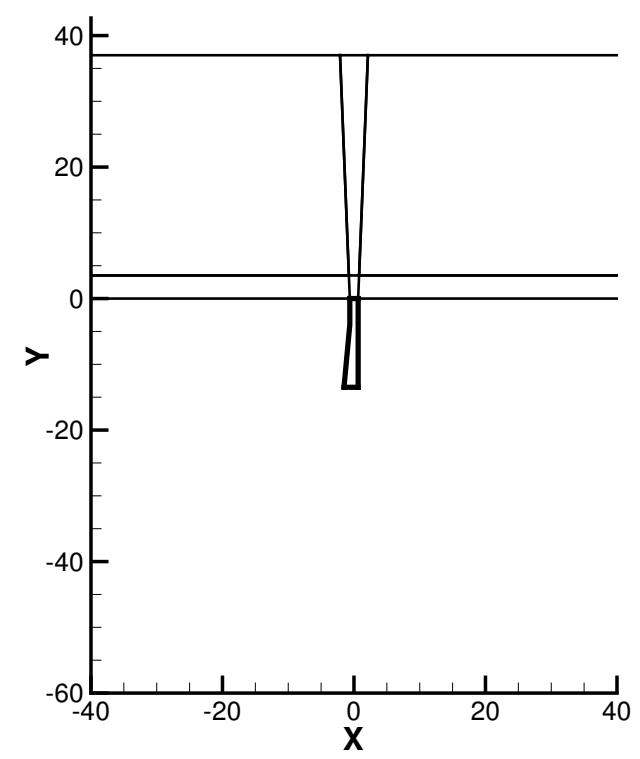

Figure 5: Simplified model of actuator 


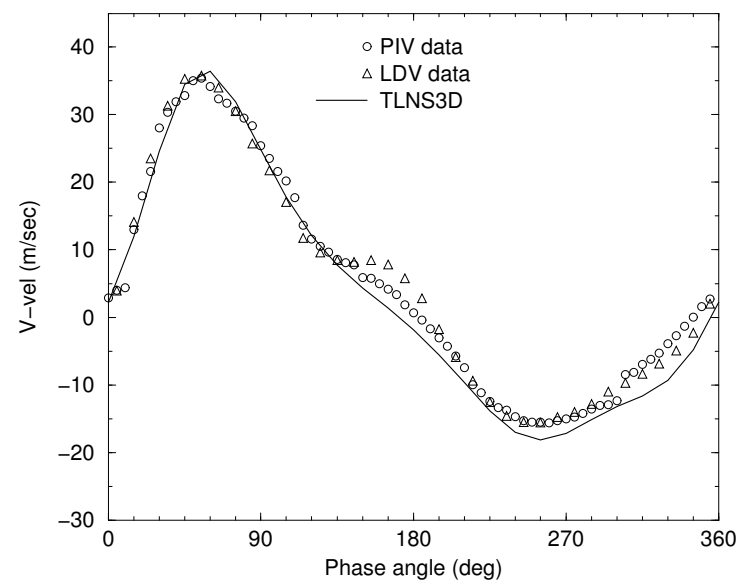

Figure 6: Time-history of v-vel near slot exit, Synjet II

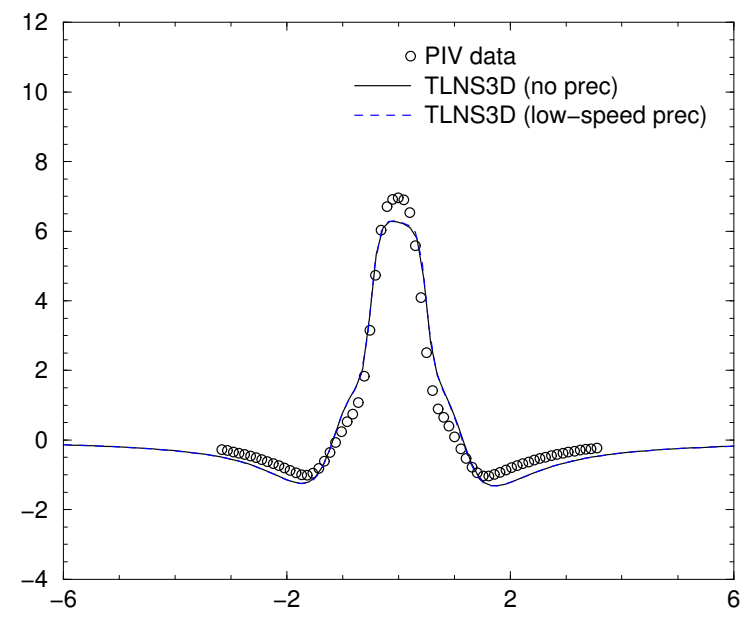

Figure 8: Average v-vel at $\mathrm{y}=1 \mathrm{~mm}$, Synjet II

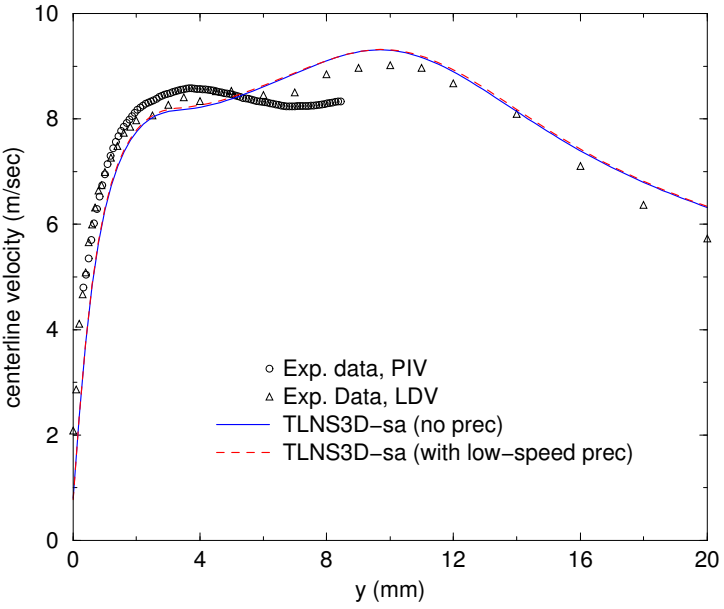

Figure 7: Average v-vel along centerline, Synjet II

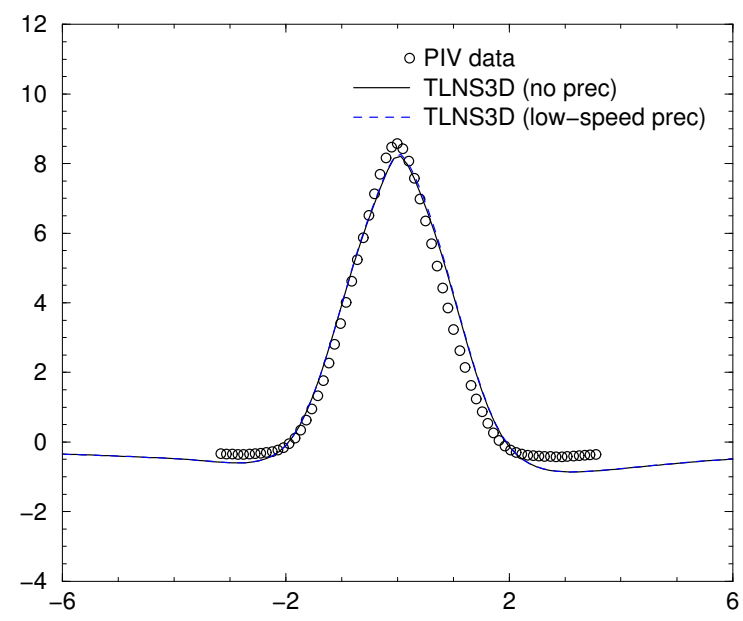

Figure 9: Average v-vel at $\mathrm{y}=4 \mathrm{~mm}$, Synjet II 


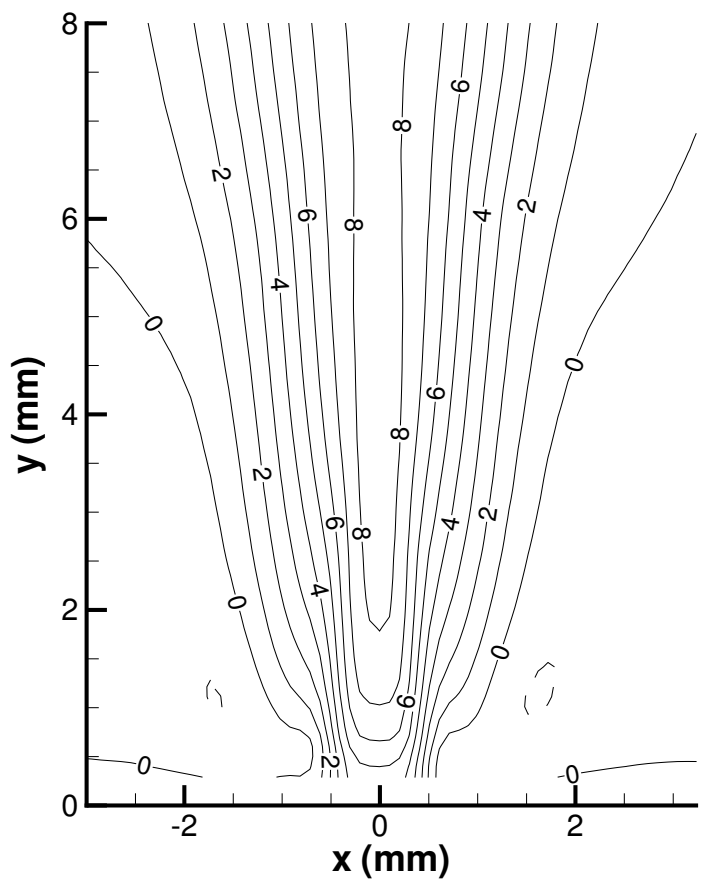

(a) PIV measurements

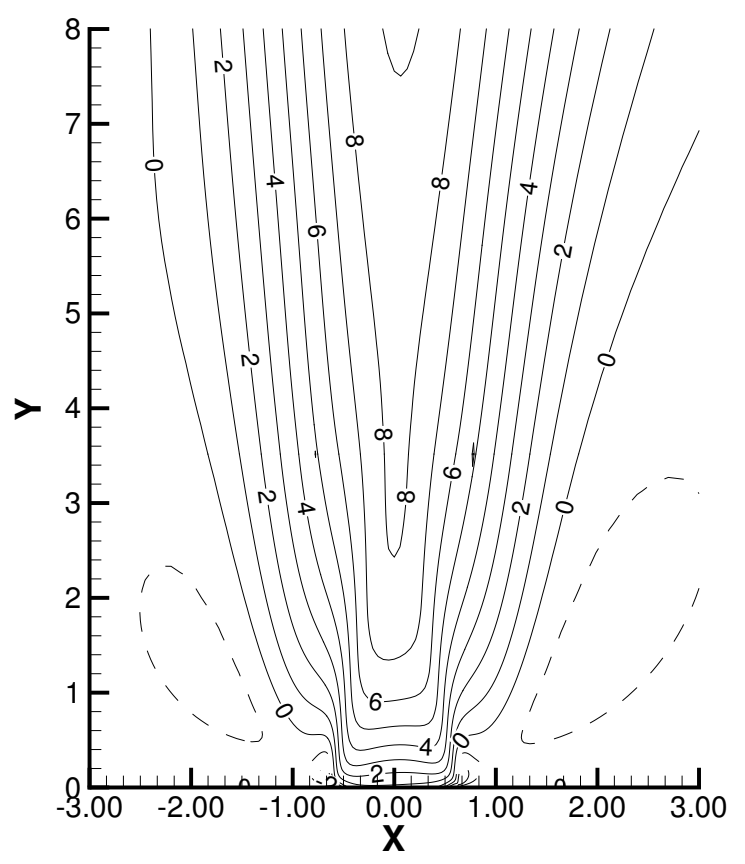

(b) TLNS3D computations

Figure 10: Time-averaged v-velocity contours for Synjet II



(a) $x=0, y=2 \mathrm{~mm}$



(b) $\mathrm{x}=0, \mathrm{y}=4 \mathrm{~mm}$

11

Figure 11: Phase-averaged v-velocity comparisons for Synjet II 




Figure 12: Contour plots of u-vel, PIV data at phase $=75^{\circ}$

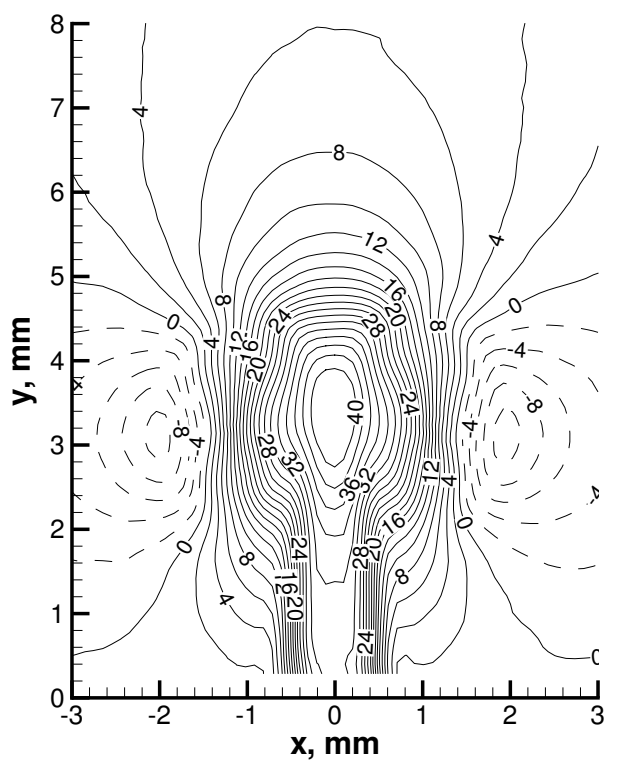

Figure 14: Contour plots of u-vel, PIV data at phase $=75^{\circ}$



Figure 13: Contour plots of u-vel, TLNS3D results at phase $=75^{\circ}$



Figure 15: Contour plots of v-vel, TLNS3D results at phase $=75^{\circ}$ 


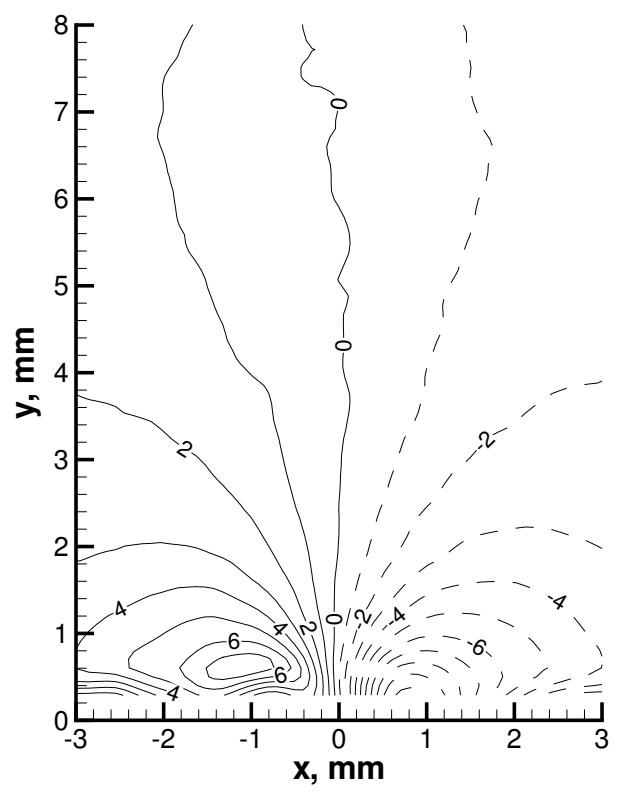

Figure 16: Contour plots of u-vel, PIV data at phase $=255^{\circ}$



Figure 18: Contour plots of u-vel, PIV data at phase $=255^{\circ}$

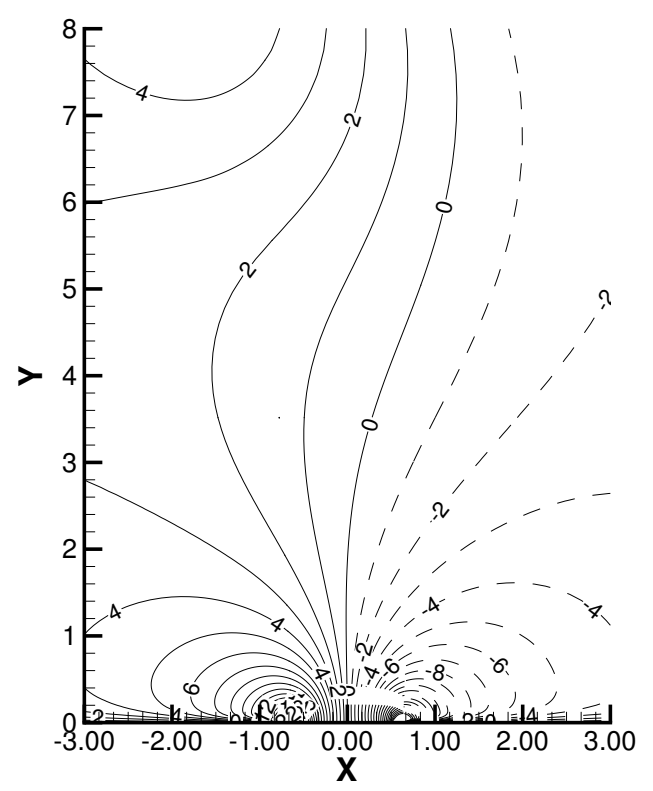

Figure 17: Contour plots of u-vel, TLNS3D results at phase $=255^{\circ}$

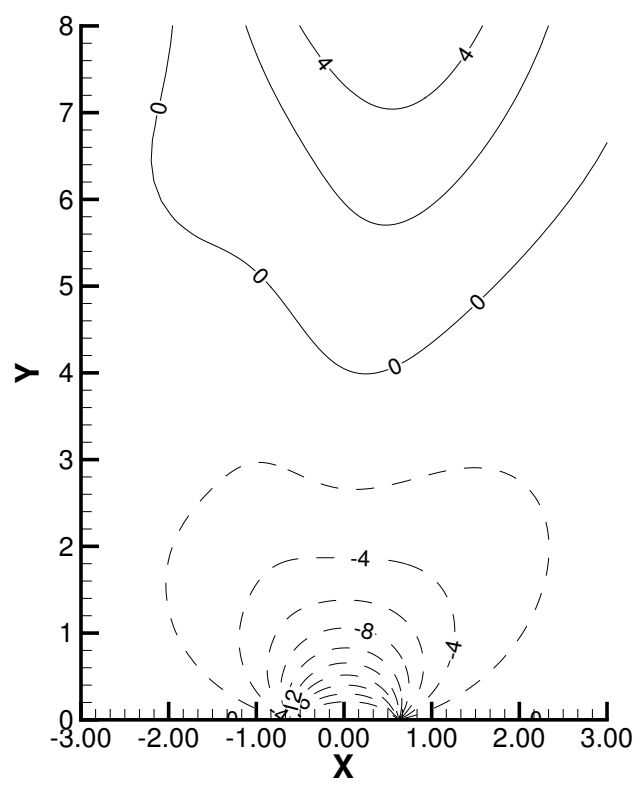

Figure 19: Contour plots of v-vel, TLNS3D results at phase $=255^{\circ}$

13 